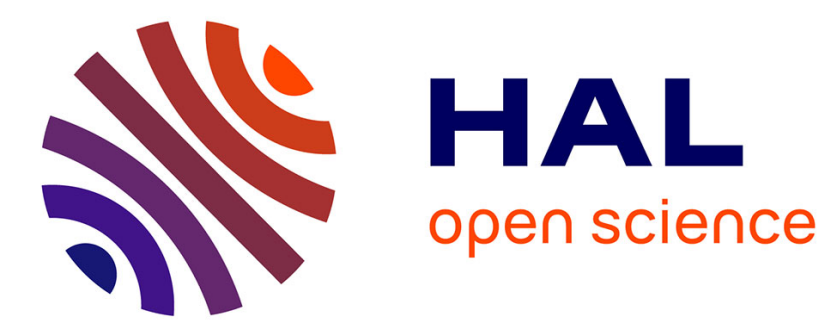

\title{
Vehicle lane keeping control based on piecewise affine regions
}

\author{
Andre Benine-Neto, Stefano Scalzi, Said Mammar
}

\section{To cite this version:}

Andre Benine-Neto, Stefano Scalzi, Said Mammar. Vehicle lane keeping control based on piecewise affine regions. 14th International IEEE Conference on Intelligent Transportation Systems (ITSC 2011), Oct 2011, Washington, United States. pp.907-912, 10.1109/ITSC.2011.6082817 . hal-00654103

\section{HAL Id: hal-00654103 https://hal.science/hal-00654103}

Submitted on 12 Jul 2021

HAL is a multi-disciplinary open access archive for the deposit and dissemination of scientific research documents, whether they are published or not. The documents may come from teaching and research institutions in France or abroad, or from public or private research centers.
L'archive ouverte pluridisciplinaire HAL, est destinée au dépôt et à la diffusion de documents scientifiques de niveau recherche, publiés ou non, émanant des établissements d'enseignement et de recherche français ou étrangers, des laboratoires publics ou privés. 


\title{
Vehicle Lane Keeping Control Based on Piecewise Affine Regions
}

\author{
André Benine-Neto ${ }^{1}$, Stefano Scalzi ${ }^{2}$, Saïd Mammar ${ }^{3}$
}

\begin{abstract}
This paper shows the design of a lane keeping steering control, that considers the nonlinear behavior of the tire-road lateral forces, by parameterizing its dynamics with respect to the yaw rate, as it is a low cost measurable variable. The proposed control strategy uses a piecewise linear (PWL) proportional double-integral derivative $\left(P I I D_{i}\right)$ control based on the lateral offset, measured by the vision system. The switches of the PWL controller are triggered by the yaw rate to take into account the nonlinear behavior of the tire forces. The stability is proved by a piecewise quadratic Lyapunov function. Simulation tests, including disturbances rejections and step references, are carried out on a standard CarSim DClass vehicle model to explore the robustness with respect to unmodelled dynamics. The simulation results confirm that the proposed PWL control can greatly improve the vehicle stability and it is advantageous in very demanding maneuvers.
\end{abstract}

\section{INTRODUCTION}

It can be noted that earlier work was devoted to autonomous vehicles in highway scenarios in which the experienced lateral accelerations are generally low [7]. These scenarios have also been considered in more recent works for the development of assistance systems which replace the driver during the lane departure avoidance maneuvers [14]. Nevertheless, in order to provide more safety to the drivers, these Lane Keeping Assistance Systems (LKAS) must be able to operate in the complete domain of tire forces, including the nonlinear behavior [9] and [6], as many accidents occur due to the vehicle loss of control when the tire forces are saturated.

A possibility to take into account the nonlinear behavior of the lateral tire forces is the design of a parallel distributed controller based on a Takagi-Sugeno model [13] and [2].

Piecewise affine (PWA) systems also offer a possibility to model the tire forces saturation and they have been used in the development of some driver assistance systems. In [5] the longitudinal tire forces are approximated by PWA functions for a traction control problem. The lateral tire forces have also been considered in PWA form to design yaw rate controllers in [4], [3] and [17].

In [3], the authors proposed a parametrization of the vehicle dynamics in terms of yaw rate to design a PWL controller for an active steering system. The parametrization in terms of this variable is chosen because sensors to measure it are available in the currently commercialized vehicles.

\footnotetext{
${ }^{1}$ IFSTTAR - LIVIC, 14 Route de la Minière, 78000 Versailles, France (e-mail: andre.benine-neto@ifsttar.fr)

2 University of Rome Tor Vergata, Electronic Engineering Department - DIE, Via del Politecnico 1, 00133 Roma - Italy, (e-mail: scalzi@ing.uniroma2.it)

${ }^{3}$ IBISC - EA 4526 EUVE, 40 rue du Pelvoux CE1455, 91020, Evry, Cedex, France (e-mail: said.mammar@inrets.fr)
}

In this paper, the same approaches are employed for the approximation of the lateral tire force and the parametrization of vehicle dynamics. Nevertheless, the model is extended with the positioning of the vehicle in the lane in order to design a lane keeping controller which takes into account the lateral tire force nonlinear tire behavior and is able to reject disturbances such as the road curvature.

The paper is organized as follows: Section II presents the vehicle lateral dynamics. In Section III, the PWA approximation of the nonlinear model is shown, as well as a parametrization to describe the partitions in terms of the yaw rate. The strategy used for the design of the Piecewise Linear (PWL) control is addressed in Section IV. The proof of stability for the closed-loop PWA system is presented in Section V. The results obtained by simulations are discussed in Section VI and a conclusion summarizes the improvements obtained by the proposed lane keeping controller design.

\section{NONLINEAR VEHICLE MODEL}

A widely used and simplified single track vehicle model, as illustrated Fig. 1, was introduced by [1] and is considered to capture the essential vehicle lateral steering dynamics:

$$
\begin{aligned}
m\left(\dot{v}_{y}+r v_{x}\right) & =f_{s f} \cos \delta_{f}+f_{s r} \\
J \dot{r} & =l_{f} f_{s f} \cos \delta_{f}-l_{r} f_{s r}
\end{aligned}
$$

where $r$ is the vehicle yaw rate, $v_{x}$ and $v_{y}$ are the longitudinal and lateral vehicle speed, $\delta_{f}$ is the steering angle, $m$ is the vehicle mass, $l_{f}\left(l_{r}\right)$ is the distance from the front (rear) axle to the center of gravity (CG), $J$ is the vehicle inertia with respect to the vertical axis through the CG and $f_{s x}$, with $x=f, r$, are the front and rear lateral forces which are modeled according to the Pacejka tire model [16].

$$
\begin{gathered}
f_{s x}\left(\alpha_{x}\right)=D \sin \left\{C \operatorname{atan}\left[(1-E) B \alpha_{x}+E \operatorname{atan}\left(B \alpha_{x}\right)\right]\right\}, \\
\alpha_{f}=\delta_{f}-\frac{v_{y}+l_{f} r}{v_{x}}, \quad \alpha_{r}=-\frac{v_{y}-l_{r} r}{v_{x}}
\end{gathered}
$$

where $\alpha_{f}\left(\alpha_{r}\right)$ is the front (rear) tire sideslip angle.

The vehicle parameters for the simplified single track vehicle model (1), and the parameters from the Pacejka tire model ( $B, C, D$ and $E$ ) (2) are presented in Table I. Their numerical values have been identified from the CarSim DClass vehicle model equipped with 225/65R17 front and rear tires.

The Pacejka tire model is depicted by the solid line of the left plot in Fig. 2.

The simple nonlinear model (1) shows, as well known in literature (see for example [15]), a limited stability region containing two unstable equilibrium points and a stable one 


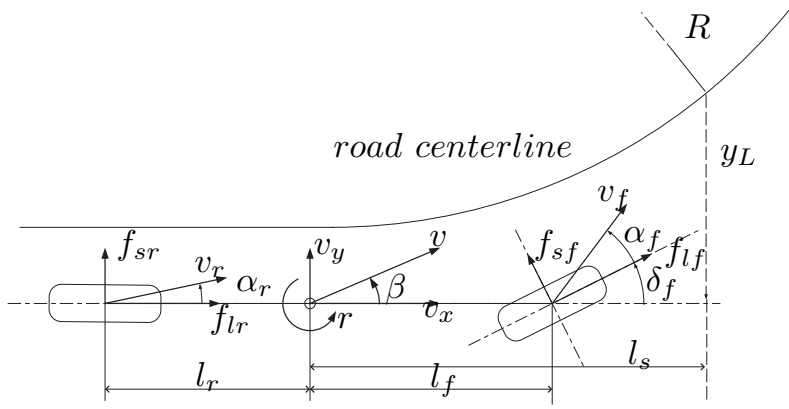

Fig. 1. Single track vehicle model
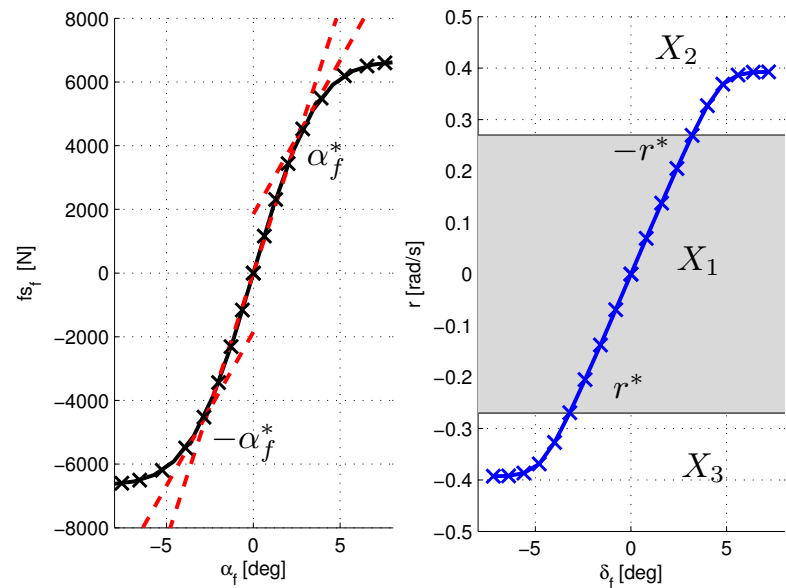

Fig. 2. Pacejka model for CarSim D-Class vehicle model equipped with 225/65R17 front and rear tires (solid line) and PWA approximations (dashed line)

TABLE I

VEHICLE PARAMETERS FOR THE LINEAR MODEL (5):

\begin{tabular}{|l|rr||l|rr|}
\hline$m$ & 2023 & {$[\mathrm{~kg}]$} & $J$ & 6286 & {$\left[\mathrm{~kg} \mathrm{~m}^{2}\right]$} \\
$l_{f}$ & 1.26 & {$[\mathrm{~m}]$} & $l_{r}$ & 1.90 & {$[\mathrm{~m}]$} \\
$a$ & 1.5 & {$[\mathrm{~m}]$} & $l_{s}$ & 6 & {$[\mathrm{~m}]$} \\
$B_{f, r}$ & 11.4592 & & $C_{f, r}$ & 1.4 & \\
$D_{f}$ & 6628 & & $D_{r}$ & 4557 & \\
$E_{f}$ & -0.5 & & $E_{r}$ & -0.7 & \\
\hline
\end{tabular}

(bifurcation analysis). which also depends on the driver steering wheel angle, The causes of the instability, which also depends on the driver steering angle, are due to the nonlinear tire behavior of the tire-road forces which are taken into account in the design of the lane keeping controller by the piecewise affine (PWA) model described in the next section.

\section{Piecewise Affine Vehicle model}

In order to approximate system (1) by a PWA system, the lateral tire forces domain, which are function of $\alpha_{f}$ and $\alpha_{r}$, can be partitioned in polyhedral sets, which are indicated by the index $i$, and for each of them an affine system is used to describe the corresponding linearized dynamics.

Considering that the front and rear tire forces described by (2) are approximated by the following PWA functions:

$$
\begin{gathered}
f_{s f}\left(\alpha_{f}\right)=e_{f i}+d_{f i} \alpha_{f} \\
f_{s r}\left(\alpha_{r}\right)=e_{r i}+d_{r i} \alpha_{r}
\end{gathered}
$$

where $d_{f i}, d_{r i}, e_{f i}$ and $e_{r i}$ depend on the front and rear tire forces for each region $(i)$.

System (1) is linearized around uniform rectilinear motion (constant $v_{x}=v, r=0, v_{y}=0, \delta_{f}=0$ ) taking into account the PWA functions (4) adopted to approximate the nonlinear tire forces. The vehicle dynamics can be represented by the PWA system:

$$
\dot{x}^{d}=A_{i}^{d} x^{d}+B_{i}^{d} u+a_{i}^{d},
$$

and the corresponding dynamics are:

$$
\begin{aligned}
& A_{i}^{d}=\left[\begin{array}{cc}
-2 \frac{d_{f i}+d_{r i}}{m v} & -1-2 \frac{d_{f i} l_{f}-d_{r i} l_{r}}{m v^{2}} \\
2 \frac{d_{r i} l_{r}-d_{f i} l_{f}}{J} & -2 \frac{d_{f i} l_{f}^{2}+d_{r i} l_{r}^{2}}{J v}
\end{array}\right], \\
& B_{i}^{d}=\left[\begin{array}{c}
\frac{2 d_{f i}}{m v} \\
\frac{2 d_{f i} l_{f}}{J}
\end{array}\right], \quad a_{i}^{d}=\left[\begin{array}{c}
2 \frac{e_{f i}+e_{r i}}{m v} \\
2 \frac{e_{f i} l_{f}-e_{r i} l_{r}}{J}
\end{array}\right] .
\end{aligned}
$$

where the control input is the front wheel steering angle $u=\delta_{f}$, while the vehicle sideslip angle $\beta$ (which replaces $v_{y}$ since $v_{y}=v \sin \beta$ ) and the yaw rate $r$ are the state variables $x^{d}=[\beta, r]^{T}$.

The partitions, indicated in the left subplot of Fig. 2 by the values of front (rear) sideslip angle $\alpha_{f,(r)}^{*}$ (only front tire is depicted for sake of clarity), must be chosen adequately in order to take into account the linear and the nonlinear tire behavior of the tire forces.

Usually the partitions of PWA systems are expressed in terms of the state-space variables, therefore in [5] and [4] system (5) is rewritten in terms of $\alpha_{f}$ and $\alpha_{r}$. However, these variables are not measurable and for that estimation algorithms and expensive optical sensors are required.

In order to circumvent this drawback, the procedure proposed in [3] is employed in this work to parametrize the vehicles dynamics, so that the partitions of the PWA system are defined only in terms of the vehicle yaw rate, which is available for measurement at low cost. The parametrization is obtained from a steady-state analysis in which (1) is solved for different values of steering angles, and the steady-state values of $v_{y}$ and $r$ are used in equations (3) to compute the front (rear) wheel sideslip angle, $\alpha_{f,(r)}$ in steady-state tire behavior.

Values of yaw rate at steady-state, which yields the wheel sideslip angle corresponding to the partitioning of the tire force domain (represented by $\alpha_{f}^{*}$ in the right plot of Fig. 2), can be used to define the partitions of the PWA system (5) in term of state-space variables. These parametrized partitions, represented by the two hyperplanes at $r=-r^{*}$ and $r=$ $r^{*}$, define the operating regions denoted by $X_{i}$, with $i=$ $\{1,2,3\}$ of the PWA system (5), as shown in Fig.2.

For the region that includes the origin (denoted by $i=$ 1 ), the commonly used linear tire forces approximation is obtained by setting $e_{f 1}=0, e_{r 1}=0, d_{f 1}=c_{f}$ and $d_{r 1}=c_{r}$ 
where $c_{f}$ and $c_{r}$ are related to the parameters in the Pacejka's formula as follows:

$$
c_{f}=B_{f} C_{f} D_{f}, \quad c_{r}=B_{r} C_{r} D_{r} .
$$

Even though the approximation of the tire force (and corresponding yaw rate parametrization) can be refined, it is shown in the simulation section that improved performance can be obtained by using this partitioning.

\section{A. Additional Dynamics for Lane Keeping}

For lane keeping purposes, the model (5) has to be expanded with the dynamics of the relative yaw angle and the lateral displacement with respect to the lane centerline. These measurements are provided by a video sensor. Let $\psi_{L}=\psi-\psi_{t}$ be the yaw angle error which is the angle between the vehicle longitudinal axis and the tangent to the road. The road reference curvature $\rho_{\text {ref }}$ is defined by $\left(\dot{\psi}_{t}=v \rho_{\text {ref }}\right)$, and the following equality can be derived:

$$
\dot{\psi}_{L}=r-v \rho_{\text {ref }} .
$$

Denoting by $l_{s}$ the look-ahead distance, the equation giving the evolution of the measurement of the lateral offset $y_{L}$ from the centerline at sensor location is obtained by

$$
\dot{y}_{L}=v\left(\beta+\psi_{L}\right)+l_{s} r .
$$

Equations (9) and (10) are included in system (5) and the PWA system describing the vehicle dynamics and positioning is described by:

$$
\dot{x}^{p}=A_{i}^{p} x^{p}+B_{i}^{p} u+B_{\rho}^{p} \rho_{r e f}+a_{i}^{p}
$$

where $x^{p}=\left[\beta, r, \psi_{L}, y_{L}\right]^{T}$ and

$$
\begin{aligned}
A_{i}^{p} & =\left[\begin{array}{cccc}
A_{i}^{d} & 0_{2 \times 2} \\
0 & 1 & 0 & 0 \\
v & l_{s} & v & 0
\end{array}\right], \\
B_{i}^{p} & =\left[\begin{array}{lll}
\left(B_{i}^{d}\right)^{T} & 0 & 0
\end{array}\right]^{T}, \\
B_{\rho}^{p} & =\left[\begin{array}{cccc}
0 & 0 & -v & 0
\end{array}\right]^{T}, \\
a_{i}^{p} & =\left[\begin{array}{lll}
\left(a_{i}^{d}\right)^{T} & 0 & 0
\end{array}\right]^{T} .
\end{aligned}
$$

The controller to ensure zero lateral offset for model (11), despite the road curvature is presented in the sequel.

\section{Control Strategy}

In order to steer the vehicle to a zero lateral-offset condition, it is necessary to model the dynamics of the road curvature $\rho_{\text {ref }}$ considering it as a disturbance on the lateraloffset. According to [18], the road curvature can be modeled as straight, circular and clothoid sections. Clothoids ensure a smooth transition between different road curvature values (e.g. straight to circular roads) and they may cover large distances on the road. For a vehicle traveling at a constant speed, the road curvature is constant in circular sections, therefore an integral term (denoted $I_{1 i}$ ) is needed to reject this disturbance. In section represented by a clothoid, $\rho_{\text {ref }}$ is considered as increasing linearly with respect to time (as

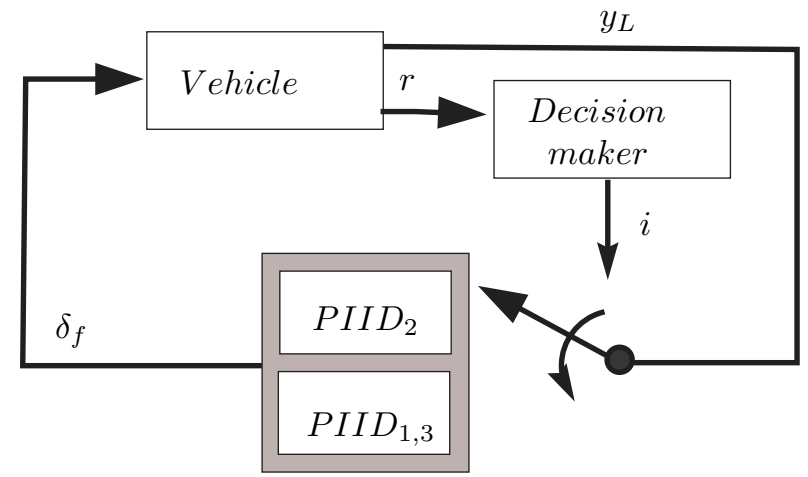

Fig. 3. Piecewise linear control schema.

a ramp function), thus an additional integral term (denoted $I_{2 i}$ ) is required to obtain zero lateral-offset. The dynamics due to the integral terms and an additional derivative term (denoted $D_{i}$ ) are defined as follows:

$$
\begin{aligned}
& \dot{\alpha}_{1}=y_{L}, \\
& \dot{\alpha}_{2}=\alpha_{1}, \\
& \dot{\alpha}_{3}=-\frac{1}{\tau} \alpha_{3}+y_{L},
\end{aligned}
$$

where $\tau$ is the filter time constant. Defining:

$$
y_{L d}=-\frac{1}{\tau^{2}} \alpha_{3}+\frac{1}{\tau} y_{L},
$$

the $P I I D_{i}$ regulator becomes:

$$
\begin{aligned}
\delta_{f} & =-P_{i} y_{L}-I_{1 i} \int_{0}^{t} y_{L} d \nu-I_{2 i} \int_{0}^{t} \int_{0}^{\nu} y_{L} d \eta d \nu-D_{i} y_{L d} \\
& =-P_{i} y_{L}-I_{I i} \alpha_{1}-I_{2 i} \alpha_{2}-D_{i} y_{L d}, \text { for } i=1,2,3 .
\end{aligned}
$$

Replacing (15) in (11), considering that $u=\delta_{f}$, and including the dynamics (13) the closed loop PWA system becomes:

$$
\dot{x}=A_{i} x+B_{\rho} \rho_{\text {ref }}+a_{i}
$$

where $x=\left[\beta, r, \psi_{L}, y_{L}, \alpha_{1}, \alpha_{2}, \alpha_{3}\right]^{T}$ and

$$
\begin{aligned}
A_{i} & =\left[\begin{array}{ccccccc}
A_{i}^{d} & 0 & -b_{1 i} k_{i} & -b_{1 i} I_{1 i} & -b_{1 i} I_{2 i} & b_{1 i} \frac{D_{i}}{\tau^{2}} \\
& & 0 & -b_{2 i} k_{i} & -b_{2 i} I_{1 i} & -b_{2 i} I_{2 i} & b_{2 i} \frac{D_{i}}{\tau^{2}} \\
0 & 1 & 0 & 0 & 0 & 0 & 0 \\
v & l_{s} & v & 0 & 0 & 0 & 0 \\
0 & 0 & 0 & 1 & 0 & 0 & 0 \\
0 & 0 & 0 & 0 & 1 & 0 & 0 \\
0 & 0 & 0 & 1 & 0 & 0 & -\frac{1}{\tau}
\end{array}\right], \\
B_{\rho} & =\left[\begin{array}{lllll}
\left(B_{\rho}^{p}\right)^{T} & 0 & 0 & 0
\end{array}\right]^{T}, \\
a_{i} & =\left[\begin{array}{llll}
\left(a_{i}^{p}\right)^{T} & 0 & 0 & 0
\end{array}\right]^{T},
\end{aligned}
$$

where $b_{1 i}=\frac{2 d_{f i}}{m v}, b_{2 i}=\frac{2 d_{f i} l_{f}}{J}$ and $k_{i}=\left(P_{i}+\frac{D_{i}}{\tau}\right)$.

Fig. 3 shows the schema for the PWL control structure.

The Simulink Response Optimization toolbox was used to tune the design parameters through numerical optimization; 
the design requirements are expressed in terms of rise time, settling time and overshoot. The gains from each $P I I D_{i}$ are obtained from a Sequential Quadratic Programming method as described in [8]. They are computed such that each subsystem is stable, however the stability of the whole systems considering the switches between each subsystem is done by means of the PWA systems theory ([10] and [11]), as described in the next section.

\section{Stability of $P I I D_{i}$ CONTRoller}

The proof of stability is based on the search of a continuous piecewise quadratic Lyapunov function by means of a convex optimization problem in terms of linear matrix inequalities (LMI). As described in [10], for the convenient treatment of the affine terms, the extended state vector $\bar{x}$ is defined as:

$$
\bar{x}=\left[\begin{array}{ll}
x & 1
\end{array}\right]^{T} .
$$

Similarly, a bar over a matrix indicates that it has been modified to be compatible with the extended state vector. Therefore the system (16) becomes:

$$
\dot{\bar{x}}=\bar{A}_{i} \bar{x}+\bar{B}_{\rho} \rho_{r e f},
$$

in which

$$
\bar{A}_{i}=\left[\begin{array}{cc}
A_{i} & a_{i} \\
0_{1 \times 7} & 0
\end{array}\right], \quad \bar{B}_{\rho}=\left[\begin{array}{c}
B_{\rho} \\
0
\end{array}\right] .
$$

\section{A. Piecewise Quadratic Lyapunov Functions}

A compact matrix parametrization of continuous piecewise quadratic functions on polyhedral partitions, named Continuity matrices, $\bar{F}_{i}=\left[\begin{array}{ll}F_{i} & f i\end{array}\right]$, is defined in [11] as:

$$
\bar{F}_{i} \bar{x}(t)=\bar{F}_{j} \bar{x}(t) \quad \text { for } \quad x(t) \in X_{i} \cap X_{j},
$$

in which $X_{i}$ and $X_{j}$ are two neighbor regions of the state space.

Then the format of the continuous piecewise quadratic Lyapunov function candidate becomes:

$$
V(x)=\bar{x}^{T} \bar{P}_{i} \bar{x}, \quad \text { for } x(t) \in X_{i}
$$

where $\bar{P}_{i}=\bar{F}_{i}^{T} T \bar{F}_{i}$, and $T$ is a symmetric matrix.

In order to express the restriction that $x \in X_{i}$ via a linear form in $x$, as required in the computations, Cell Bounding matrices, $\bar{E}_{i}=\left[\begin{array}{ll}E_{i} & e_{i}\end{array}\right]$, are defined in [11], as follows:

$$
\bar{E}_{i} \bar{x} \geq 0 \quad \text { if } \quad x(t) \in X_{i}
$$

The stability of the PWA model (16) can be proved using the theorem proposed in [10], as follows:

Consider symmetric matrices $T, U_{i}$ and $W_{i}$ such that $U_{i}$ and $W_{i}$ have non negative entries, while

$$
P_{i}=F_{i}^{T} T F_{i}, \quad \bar{P}_{i}=\bar{F}_{i}^{T} T \bar{F}_{i}
$$

satisfy:

$$
\begin{gathered}
\begin{cases}A_{i}^{T} P_{i}+P_{i} A_{i}+E_{i}^{T} U_{i} E_{i}<0 & \text { for } i=1 \\
P_{i}-E_{i}^{T} W_{i} E_{i}>0 & \end{cases} \\
\begin{cases}\bar{A}_{i}^{T} \bar{P}_{i}+\bar{P}_{i} \bar{A}_{i}+\bar{E}_{i}^{T} U_{i} \bar{E}_{i}<0 & \text { for } i=2,3 \\
\bar{P}_{i}-\bar{E}_{i}^{T} W_{i} \bar{E}_{i}>0 & \text { for }\end{cases}
\end{gathered}
$$

then, every trajectory $x(t)$ of the PWA model (16) with $\rho_{\text {ref }} \equiv 0$ tends to zero exponentially.

Solving the LMI problem ((25) and (26)) a piecewise quadratic Lyapunov function (22) has been computed proving the stability of (16), which ensures that zero lateral offset in steady state is obtained with the proposed PWL controller.

The performance of the lane keeping controller (15) is evaluated by simulations on the Carsim environment, as shown in the next section.

\section{Simulation Results}

Simulations have been carried out to validate the proposed control system with respect to nonlinear combined lateral and longitudinal tire forces and additional dynamics, such as pitch, roll and driver dynamics, which were neglected at the control design stage. The standard CarSim D-Class vehicle, used to analyze the responses of the controlled vehicles, has a nonlinear second order speed depending rack and pinion ratio steering system; for the active steering a realistic actuator with a bandwidth of $10 \mathrm{~Hz}$ is considered (see [12]). The simulations were performed at $v=30.6[\mathrm{~m} / \mathrm{s}]$.

The same vehicle in two different configurations has been used in the simulations. The first configuration consisted of the vehicle equipped with the designed controller in the linear region only (equation (15) with $i=1$ ), named lin.-ctrl; The second configuration consisted of the vehicle equipped with the proposed PWL control strategy $\left(P I I D_{i}\right)$ (equation (15) with $i=1,2,3)$, named $P W L$-ctrl. Two different scenarios are presented in the sequel: response to sudden disturbances as external lateral force and yaw moment, and response to sudden direction changes (in dry and wet pavement).

\section{A. Sudden Disturbances}

To analyze the improved performance, the vehicle has been subjected to a sudden disturbances on lateral force and yaw moment as shown in the upper-left subplot of Fig. 4. The disturbances are strong enough to make the vehicle lateral dynamics enter the nonlinear region during the lane departure avoidance maneuver, as it can be seen in the bottom-left subplot of Fig. 5, showing the active regions of the $P W L$ ctrl vehicle. It is important to note that the control input of the PWL controller is reduced when the vehicle yaw rate exits the linear region $(i=1)$, as shown in the bottomright subplot of Fig. 5. Consequently the tire sideslip angles, depicted on the upper-right subplot of Fig. 5 do not increase as much as for the vehicle lin.-ctrl. In the upper-left subplots of Fig. 4 and Fig. 5 it is shown the vehicles trajectories and the lateral-offset respectively, The reduction in about $50 \%$ of the overshot in lateral-offset for the PWL-ctrl confirms its improved performance for lane keeping purposes.

\section{B. Sudden Change in Direction}

Several maneuvers have been performed to analyze the response of the lane keeping controller with respect to change in directions. The results presented correspond to a demanding lane change maneuver, in which the vehicles reach a lateral acceleration of $8.9\left[\mathrm{~m} / \mathrm{s}^{2}\right]$, in two different 

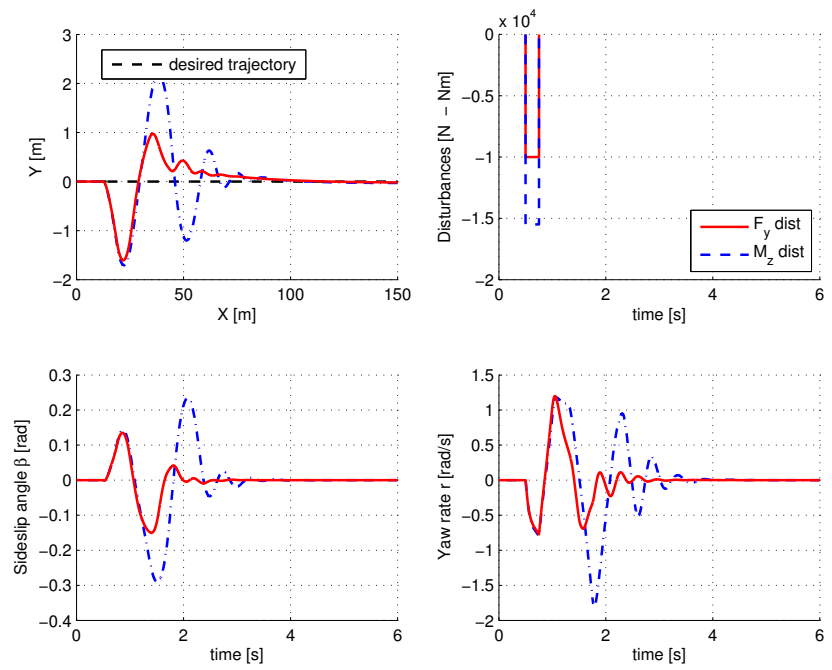

Fig. 4. Response to a sudden disturbance on vehicles lin.-ctrl (dashed line) and $P W L$-ctrl (continuous line) at $\mathrm{v}=30.6[\mathrm{~m} / \mathrm{s}]$.
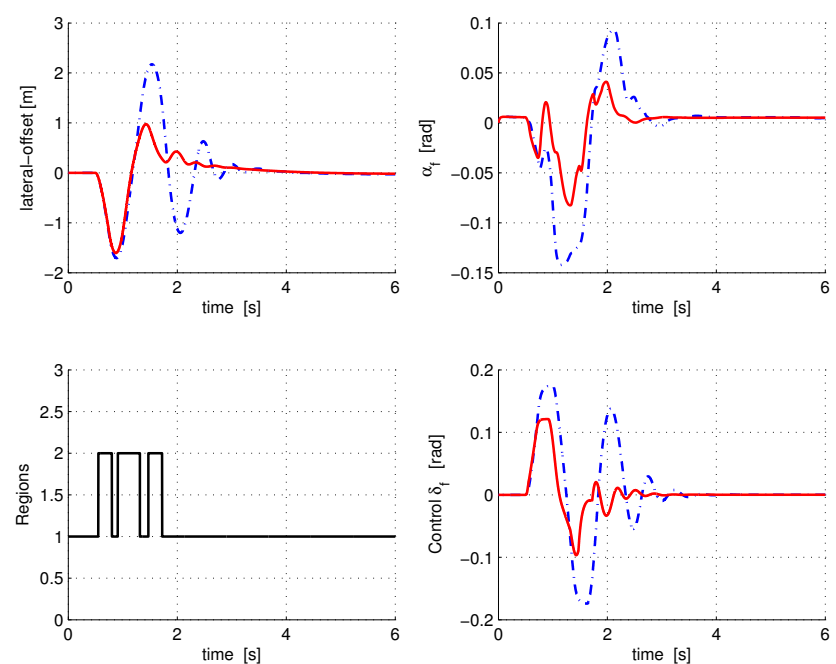

Fig. 5. Response to a sudden disturbance on vehicles lin.-ctrl (dashed line) and $P W L-\operatorname{ctrl}($ continuous line) at $\mathrm{v}=30.6[\mathrm{~m} / \mathrm{s}]$.

road adherence conditions, while no external disturbances are applied.

Considering firstly good adherence conditions, characterized by the road adhesion coefficient $(\mu=1)$, the enhanced performance of the proposed control strategy for lake keeping is clear when compared to the response of lin.$c t r l$, for the maneuver shown in the upper-left subplot of Fig. 6. Analyzing Fig 7, it can be seen that by the switch of $P W L$-ctrl between the regions $i=1,2$, in the bottomleft subplot, the control action depicted on the bottom-right, is reduced when the vehicle yaw rate reaches the partition limits. Consequently the vehicle remains in a controllable condition avoiding the saturation of tire forces which can be seen by the smaller values of front wheel sideslip angle in the upper-right subplot. Moreover the lateral-offset is steered to zero faster than for the lin.-ctrl vehicle. The vehicle lateral
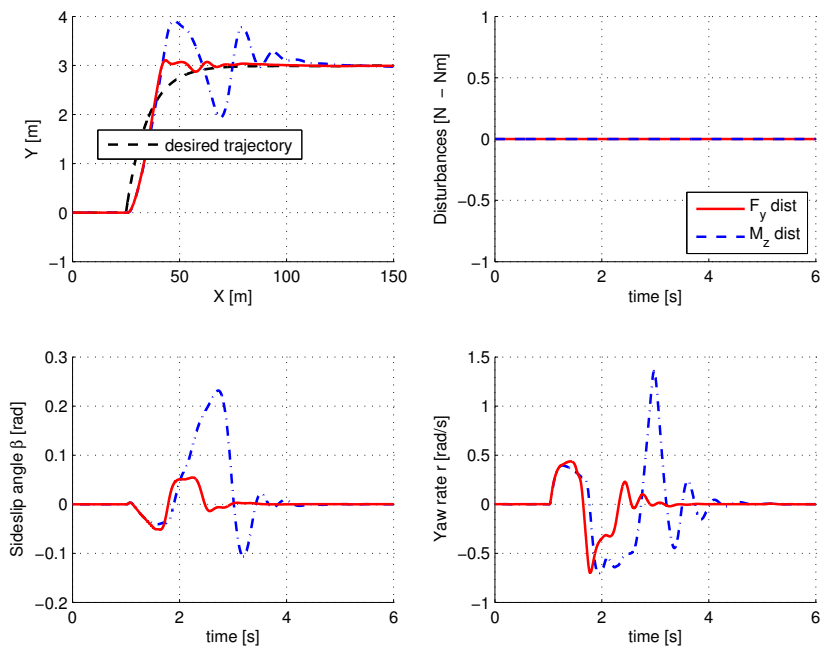

Fig. 6. Response to a lane change on vehicles lin.-ctrl (dashed line) and $P W L$-ctrl (continuous line) at $\mathrm{v}=30.6[\mathrm{~m} / \mathrm{s}]$ on dry road.
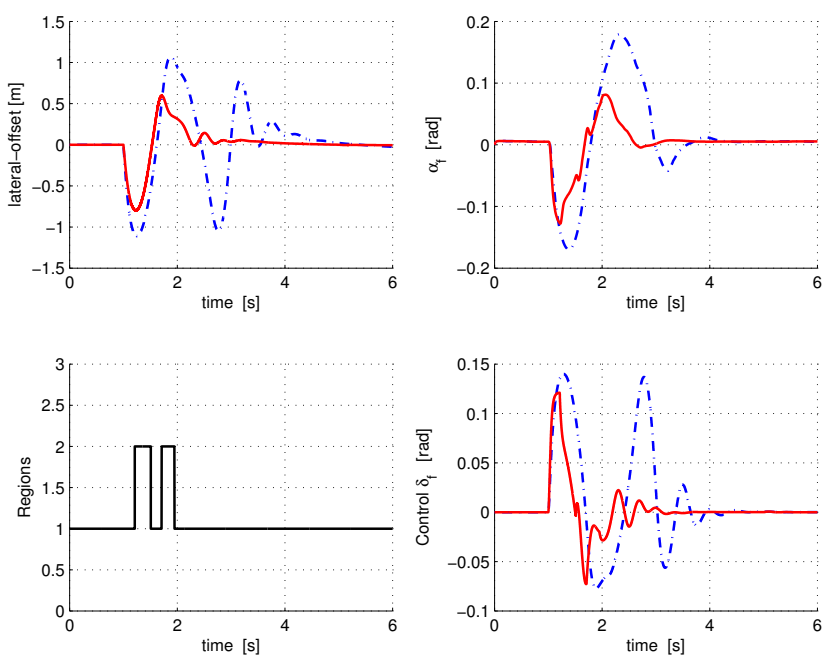

Fig. 7. Response to a lane change on vehicles lin.-ctrl (dashed line) and $P W L$-ctrl (continuous line) at $\mathrm{v}=30.6[\mathrm{~m} / \mathrm{s}]$ on dry road.

dynamics (vehicle sideslip angle and yaw rate) are shown in the bottom subplots of Fig. 6. In order to evaluate the robustness with respect to different road adherence coefficients, the same maneuver has been performed for a deteriorated road adherence $(\mu=0.7)$, corresponding to wet pavement. The results are shown in Fig. 8 and 9. Even though the lateral-offset reaches more significant values, as the maneuver is very demanding, it can be seen that, also in this case, the proposed PWL controller performance is improved with respect to the lin.-ctrl, which is not able to follow the maneuver.

\section{Conclusions}

In this paper it has been shown that a lane keeping steering control can be designed taking into account the nonlinear behavior of the tire forces. The vehicle dynamics has been parametrized with respect to the measurable yaw 

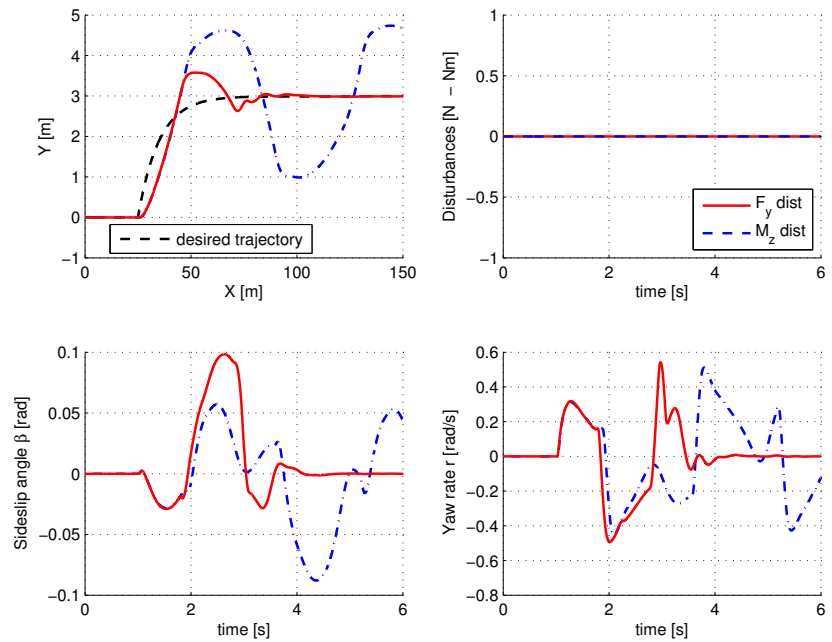

Fig. 8. Response to a lane change on vehicles lin.-ctrl (dashed line) and $P W L$-ctrl (continuous line) at $\mathrm{v}=30.6[\mathrm{~m} / \mathrm{s}]$ on wet road.
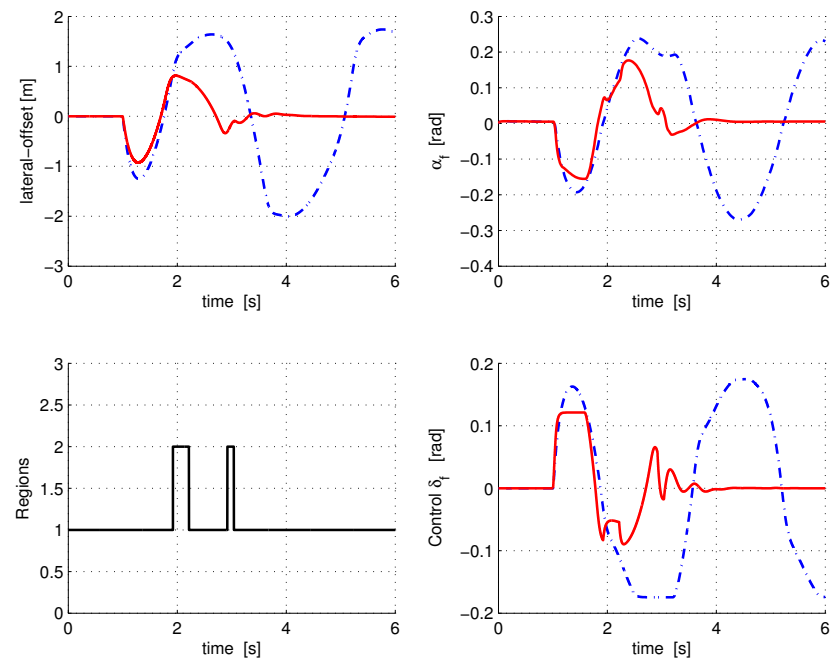

Fig. 9. Response to a lane change on vehicles lin.-ctrl (dashed line) and $P W L$-ctrl (continuous line) at $\mathrm{v}=30.6[\mathrm{~m} / \mathrm{s}]$ on wet road.

rate, considering the steady state behavior of the vehicle. This parametrization avoids the use of the tire sideslip angles which are not directly measurable, requiring estimation algorithms or high cost optical sensors. The use of the proposed $P I I D_{i}$ controller, with gains depending on the parametrized vehicle dynamics, is able to track the lane despite affine disturbances and parameters uncertainties. The stability of the controlled system is proved by the quadratic piecewise Lyapunov function, computed from a LMI problem. Simulation results including disturbances rejections and step references, carried out on a standard CarSim D-Class vehicle model, were presented to show the system enhanced performance and robustness. The simulations also confirm that the proposed PWL control, can greatly improve the vehicle stability and is advantageous in very demanding maneuvers, when compared to the linear controller, consisting in the use of the single controller as designed for the linear region only.
Moreover, the simulations showed that the switching between the PWL controllers based on a yaw rate threshold value is satisfactory and that it is able to enhance the performance, avoiding refinements of the proposed partitioning. Since this controller was designed for a constant speed, a gain scheduling scheme should be proposed in order to allow larger longitudinal speed variations. Among future work, it is also envisaged to implement the system on a prototype vehicle for perform practical evaluation.

\section{ACKNOWLEDGMENT}

This work has been sponsored by the french ANR consortium PARTAGE.

\section{REFERENCES}

[1] J. Ackermann, Robust Control. London: Springer, 2002.

[2] N. Ait Oufroukh, A. Benine-Neto, Z. Yacine, S. Mammar, and S. Glaser, "Invariant set based vehicle handling improvement at tire saturation using fuzzy output feedback," in Intelligent Vehicles Symposium (IV), 2011 IEEE, june 2011, pp. $1104-1109$.

[3] A. Benine-Neto, S. Scalzi, M. Netto, S. Mammar, and W. PasillasLepine, "Vehicle yaw rate control based on piecewise affine regions," in Proceedings of the IEEE Intelligent Vehicles Symposium, San Diego, USA, June 2010, pp. 20-25.

[4] D. Bernardini, S. D. Cairano, A. Bemporad, and H. Tseng, "Driveby-wire vehicle stabilization and yaw regulation: A hybrid model predictive control design," in Joint 48th IEEE Conference on Decision and Control and 28th Chinese Control Conference, Shanghai, P.R. China, December 2009, pp. 7621-7626.

[5] F. Borrelli, A. Bemporad, M. Fodor, and D. Hrovat, A Hybrid Approach to Traction Control. Springer Berlin / Heidelberg pp. 162174, 2001.

[6] P. Falcone, F. Borrelli, J. Asgari, H. Tseng, and D. Hrovat, "Predictive active steering control for autonomous vehicle systems," Control Systems Technology, IEEE Transactions on, vol. 15, no. 3, pp. 566580, may 2007.

[7] R. Fenton, G. Melocik, and K. Olson, "On the steering of automated vehicles: Theory and experiment," Automatic Control, IEEE Transactions on, vol. 21, no. 3, pp. 306-315, jun 1976.

[8] P. E. Gill, W. Murray, and M. H. Wright, Practical optimization. London, UK: London: Academic Press, 1981.

[9] C. Hatipoglu, U. Ozguner, and K. Redmill, "Automated lane change controller design," Intelligent Transportation Systems, IEEE Transactions on, vol. 4, no. 1, pp. 13-22, mar 2003.

[10] M. Johansson and A. Rantzer, "Computation of piecewise quadratic lyapunov functions for hybrid systems," IEEE Transactions on Automatic Control, vol. 43, no. 4, pp. 555-559, 1998.

[11] M. Johansson, Piecewise Linear Control Systems. Springer, 2002.

[12] C. MacAdam, "Application of an optimal preview control for simulation of closed-loop automobile driving," IEEE Trans. on Systems, Man, and Cybernetics, vol. 11, pp. 393-399, June 1981.

[13] S. Mammar, N. Minoiu-Enache, S. Glaser, B. Lusetti, and A. BenineNeto, "Lane keeping automation at tire saturation," in Proceedings of the IEEE American Control Conference, Baltimore, June 2010, pp. 6466-6471.

[14] N. Minoiu-Enache, M. Netto, S. Mammar, and B. Lusetti, "Driver steering assistance for lane departure avoidance," Control Engineering Practice, vol. 17, no. 6, pp. 642 - 651, 2009. [Online]. Available: http://www.sciencedirect.com/science/article/B6V2H-4V4622T1/2/1fb5831b0eacb8d63f8e78164f112d5c

[15] E. Ono, S. Hosoe, H. D. Tuan, and S. Doi, "Bifurcation in vehicle dynamics and robust front wheel steering control," IEEE Trans. on Control System Technology, vol. 6, no. 3, pp. 9-14, May 1998.

[16] H. Pacejka, Tire and Vehicle Dynamics. Elsevier ButterworthHeinemann, 2004.

[17] G. Palmieri, M.Baric, and F. Borrelli, "Constrained robust design of lateral vehicle dynamics control systems," in Proceeding of AVEC, aug 2010.

[18] R. Rajamani, Vehicle Dynamics and Control. Springer, 2006, ch. 2 Lateral Vehicle Dynamics, pp. 43-46. 